\title{
Innovations in
}

Insurance, Risk- and Asset Management

\section{Kathrin Glau}

Daniël Linders

Aleksey Min

Matthias Scherer

Lorenz Schneider

Rudi Zagst

editors 


\title{
Chapter 1
}

\section{Behavioral Value Adjustments for Mortgage Valuation}

\author{
M. Bissiri* and R. Cogo \\ Cassa Depositi e Prestiti S.p.A. \\ Rome, Italy \\ *matteo.bissiri1@gmail.com
}

\begin{abstract}
Behavioral risk affects the pricing of assets and liabilities with embedded prepayment/extension options whenever the option holder does not act purely on the strength of financial convenience but follows an uncertain and sub-optimal exercise strategy, if seen from the viewpoint of the option seller. Such behavior is particularly relevant for mortgage valuation, since mortgage prepayments are clearly influenced by exogenous and individual factors besides financial reasons. In this paper we apply the general framework, proposed by Bissiri and Cogo, for modeling behavioral risk to the particular case of the valuation of a fixed-rate mortgage portfolio. We also extend the formulas by considering a pool of heterogeneous mortgagors, leading to the introduction of specific behavioral risk adjustments ( $\beta \mathrm{VA})$ in the pricing formulas.
\end{abstract}

Keywords: behavioral risk, prepayment, mortgage, MBS, RMBS, XVA, embedded option, OAS.

\section{Introduction}

Many financial instruments are characterized by the presence of embedded options, which allow one of the counterparts to terminate the contract before maturity or modify contractual conditions, according to clauses specified at the inception. We do not refer here to the so-called automatic options, such as a cap or floor on an interest rate, but rather to options that require a decision to be taken by the option holder, such as prepayment or switch options.

On the asset side, the most typical example are mortgage loans, whose valuation is the focus of this paper. In many countries mortgagors are allowed to cancel a debt at any time by paying back the outstanding notional without any penalty (see, e.g., Davidson and Levin [1]). Prepayments also influence the pricing of mortgage backed securities (MBS), callable bonds, retail loans, loan commitments, etc. Liabilities can also embed explicit or implicit options, like in the case of puttable bonds or non-maturity deposits, which can be withdrawn by the investor at any time (see, e.g., Castagna

Open Access chapter published by World Scientific Publishing Company and distributed under the terms of the Creative Commons Attribution-NonCommercial-NoDerivatives (CC BY-NC 4.0) License. 
and Fede [2] for a review of these instruments). Insurance policies may also incorporate complex prepayment features.

Due to the presence of behavioral risk, the valuation of instruments with embedded options can be an extremely challenging task. This occurs whenever the option holder does not act purely on the strength of financial convenience but follows a sub-optimal and uncertain exercise strategy, if seen from the perspective of the option seller. Various reasons can be invoked to explain this behavior, such as a different valuation and/or modeling of the underlying contract, no financial interest in early exercise, regulatory constraints, the presence of large transaction costs, the impact of exogenous factors or, as in the case of retail customers, the lack of information or sophistication. According to a more rigorous and quantitative definition, provided by Bissiri and Cogo [3], behavioral risk can be identified with the additional source of uncertainty in the time and amount of future cash flows that an option seller faces, due to the unpredictable exercise strategy followed by the option holder.

In this paper we reconsider the impact of prepayment features ion the valuation of a mortgage portfolio from the lender's viewpoint. For this purpose, we apply the general pricing approach for contracts affected by behavioral risk, which was recently developed by Bissiri and Cogo [4]. In particular, we examine the pricing of a basket of fixed-rate mortgages, issued at different times and with different contractual rates. We also extend pricing formulas to the case of a heterogeneous pool of mortgagors, with different creditworthiness and prepayment behavior. The aim is to develop a coherent and flexible enough framework, that can be tailored and calibrated to specific instruments depending on available data.

Clearly, mortgage valuation depends also on credit risk (i.e. the borrower is unable to repay the loan), although mitigated by the underlying asset acting as collateral (e.g. the house property). For instance, the recent sub-prime crisis in the US market was caused by large-scale mortgagor defaults. However, a detailed modeling of default risk is out of scope of the present paper.

Nonetheless, the approach used to model mortgage prepayments takes advantage of a full parallel with credit risk modeling and combines the features of option-based and intensity models reported in literature. A particular emphasis is placed on the calibration of the risk premium. Since exercise decisions taken by irrational option holders are influenced at the same time by financial, exogenous and individual reasons, behavioral risk clearly has a hybrid nature. However, whilst it is possible to implement a 
replicating strategy to hedge (or significantly reduce) portfolio sensitivity to financial risk factors, exogenous or idiosyncratic risk can only be diversified in a large portfolio. Therefore, a mixed approach seems the most appropriate choice for pricing purposes. A market risk premium for all financial risk factors is inferred from the quotes of liquid traded instruments, which could be used to set up a replicating strategy. In contrast, a traditional risk charge is calculated in order to compensate for unexpected losses arising from other sources of risk, leading to the definition of behavioral value adjustment ( $\beta \mathrm{VA})$.

The paper is divided into three sections: $(i)$ Firstly, the literature about mortgage valuation is summarized briefly; (ii) Secondly, the general pricing framework proposed by Bissiri and Cogo [4] and its underlying hypotheses are described in more detail; (iii) Finally, a pricing formula for a basket of fixed-rate mortgages is derived.

\section{Literature review}

The valuation of baskets of mortgages has been studied since early 1980s and several pricing approaches have been proposed.

Econometric models assume that prepayment rates are dependent variables, and functions of a set of explanatory variables. Although they potentially allow a detailed description of a debtor's behavior by means of a large set of regressors, econometric models are less practical for pricing purposes, since risk-neutral dynamics have to be specified for all risk factors.

Option-based models were introduced along with the development of the no-arbitrage pricing theory for derivatives. In order to account for deviations from rationality, exercise constraints or transaction costs are introduced as in the models of Dunn and McConnell [5], Stanton [6], Longstaff [7], Kalotay et al. [8], Davidson and Levin [9]. Conditionally to a specific market scenario, the strategy followed by the option holder is still deterministic but sub-optimal, if seen by the option seller. Although option-based models permit valuations that are theoretically consistent with prepayment scenarios, their use seems to be a more natural choice only if prepayments are almost rational from a financial standpoint.

Intensity-based models have been proposed as a valid alternative by several authors, such as Schwartz and Torous [10], Deng et al. [11], Kau et al. [12], Kwok et al. [13], Consalvi and Scotto di Freca [14] and Chernov et al. [15]. They exploit the similar methodology widely applied in credit risk modeling. Like default probabilities, prepayment probabilities 
are stochastic variables, depending on a set of risk drivers. Intensity models naturally incorporate exogenous sources of risk, accounting for the residual variability in prepayment rates not determined by financial reasons. Our understanding is that intensity-based models are particularly suitable for those situations where early prepayments mainly depend on exogenous factors rather than on financial variables.

Finally, hybrid models have recently been proposed by combining the features of different approaches, such as in the works of Goncharov [16], Castagna and Fede [2], Kolbe and Zagst [17], [18]. For instance, Kolbe and Zagst [17] have generalized the traditional proportional hazard approach by developing and calibrating an extended hybrid model. Mortgage prepayment rates are explained in terms of a combination of market and non-financial drivers, like, e.g., gross-domestic-product (GDP).

All models reported in literature have to be calibrated with historical data. It is important to stress that mortgage prepayments are a very complex phenomenon. Despite the model family and regardless of its complexity, there are always some latent risk factors not captured by the model, as demonstrated by the fit residuals, which result after the calibration process. A similar issue is found when comparing theoretical model prices to the market quotes of liquid RMBS. Typically, an option-adjusted-spread has to be applied to discount factors in order to obtain a perfect match (see, e.g., Davidson and Levin [1] or Gabaix et al. [19]). Such residual dispersion cannot be neglected, since it may contribute significantly to the variability in the future cash flows of a mortgage portfolio. In this paper we model it as an exogenous error process, leading to a prudential correction in the pricing formula. In our opinion, such an approach is particularly suitable for calibrating the risk premium in the absence of liquid market benchmarks.

\section{A general framework for modeling behavioral risk}

In this section we summarize the general framework recently proposed by Bissiri and Cogo [4] for the pricing of financial products with embedded options in the presence of behavioral risk. The approach is intended to be general and adaptable to model the behavior of different categories of investors and to price different types of financial instruments.

It takes advantage of the similarities with credit risk modeling. For a review of interest and credit models see e.g. the books of Brigo and Mercurio [20] or Schönbucher [21]. 


\subsection{Defining behavioral risk}

Although there is no unanimous consensus about a single definition, behavioral risk, as well as all sources of risk, can always be identified as the additional source of uncertainty in the future cash flows of a contract that it generates. More precisely, it can be identified with the additional source of uncertainty in the time and amount of future cash flows that an option seller faces, due to the unpredictable exercise strategy followed by the option holder, see Bissiri and Cogo [3], [4].

In a world where all investors are fully rational from a financial standpoint and agree on a no-arbitrage model for market factors, the optimal exercise strategy for the prepayment option is uniquely determined.

In the particular case of a mortgage with no prepayment penalties, the optimal exercise time, which maximizes the return for the option holder, occurs whenever the redemption value (i.e. the outstanding balance of the loan) falls below the so-called continuation value (i.e. the present value of all future installments). ${ }^{1}$ Conditional to a specific market scenario of mortgage rates, cash flows are completely deterministic.

In contrast, only a probability for option exercise can be estimated under the presence of behavioral risk. More importantly, cash flows are uncertain and cannot be predicted purely on the strength of financial convenience. Historical aggregate prepayment rates relative to baskets of mortgages imply that some debtors prepay when this is not convenient and some others do not prepay even if this is convenient. Typically, a S-shaped dependence from the rate shift ${ }^{2}$ is observed (see, e.g., Peristiani [22]).

Thus, empirical evidence demonstrates that prepayments must be influenced by exogenous and/or individual reasons besides financial factors. Such behavior results in an additional variability in the future cash flows, even subject to a particular market scenario. More precisely, behavioral risk can be associated with the conditional variance which arises whenever the option holder does not follow an optimal exercise strategy as seen from the option seller's perspective.

\footnotetext{
${ }^{1}$ In practice, such condition is met as soon as mortgage rates prevailing in the market decrease so that it is possible to refinance the debt at lower cost.

${ }^{2}$ The rate shift can be defined as the difference between the mortgage contractual rate and the new rate prevailing in the market at a point in time.
} 


\subsection{A general framework in parallel with credit risk}

Exercise probabilities, $Q_{i}(t)$, like default probabilities, are modeled as stochastic variables, depending on both market $X(t)$ and exogenous factors $Z(t)$.

$$
\ln \left[Q_{i}(t)\right]=R_{i}(t, X(t), Z(t) ; \theta(t))
$$

where $R_{i}$ is a generic response function of the $i$-th mortgagor and $\theta(t)$ is a set of model parameters.

Potentially, the responsiveness to changing market conditions of two different debtors may vary significantly. It is well known that heterogeneity plays a crucial role in determining the aggregate prepayment rate of a basket of mortgages and can be responsible, at least to some extent, for the socalled burnout effect, namely, the tendency of prepayment rates to decline over the lifetime of a contract.

Financial factors, $X$, include all market variables that impact on contractual payments and are required for the valuation of the full rational cost of the option. For instance, in the case of mortgages, interest rates and credit spreads (or default probabilities) should be taken into account.

In principle, a large set of economic or individual observables can be selected in order to explain exercise rates besides financial factors, such as gross-domestic-product, unemployment rate, personal income, age, etc. By adopting a reduced-form approach, we introduce generic exogenous drivers, $Z$, orthogonal to the financial ones and characterized by a systemic component (i.e. common to all investors) and a purely idiosyncratic one.

Conditional independence is also assumed. Subject to the realization of a scenario of all risk factors $\{X, Z\}$, prepayment decisions are taken independently by each single mortgagor. In addition, if a debtor holds several positions, the exercise decisions of options embedded in different contracts are independent from each other, subject to the same risk scenario. ${ }^{3}$

Mathematically,

$$
\mathbb{V}\left[\mathbb{I}\left(\tau_{p}^{i}>t_{k}\right), \mathbb{I}\left(\tau_{q}^{j}>t_{h}\right) \mid X, Z\right]=0 \quad \text { for } i \neq j \text { or } p \neq q
$$

\footnotetext{
${ }^{3}$ For example, let us consider a borrower holding two distinct mortgages A e B with contractual fixed rates of $1 \%$ and $5 \%$, respectively, when prevailing market rates are around $3 \%$. The exercise probability of two loans will satisfy the inequality $Q_{A}<<Q_{B}$, if computed through a reasonable specification of (1). However, subject to that particular scenario, exercise decisions are taken independently. It is worth noting that in typical residential mortgage portfolios most mortgagors hold a single contract.
} 
A further hypothesis consists in assuming that exogenous factors follow a mean-reverting process, so that their effect tends to vanish over a long period of time. Without loss of generality, we can reformulate the problem by taking advantage of conditional probabilities and set

$$
R_{i}(t, X(t), Z(t) ; \theta(t))=\phi_{t}(X(t) ; \theta(t))+\eta_{t}(Z(t) \mid X(t) ; \theta(t))
$$

The function $\eta_{t}$ can be interpreted as a sort of error process which quantifies the variability in option exercise frequencies around the historical average, $\phi_{t}$, conditionally to a particular scenario of market factors.

Since we expect that such deviations tend to cancel out over a long observation period, we impose that $\eta_{t}$ follows a mean-reverting process with an asymptotic distribution with zero mean and finite variance.

$$
\left\{\begin{array}{l}
\mathbb{E}_{Z}^{P}\left[\eta_{\infty} \mid X\right]=0 \\
\mathbb{V}_{Z}^{P}\left[\eta_{\infty} \mid X\right] \approx s_{\infty}^{2}(X)
\end{array}\right.
$$

where variance $s_{\infty}^{2}$ is the empirical conditional variance. ${ }^{4}$ Refer to the paper by Bissiri and Cogo [4] for more details.

\subsection{Behavioral risk adjustments}

A mixed-approach has been proposed by Bissiri and Cogo [4] for computing behavioral risk premium in the absence of a market benchmark.

On the one hand, risk-neutral dynamics $(Q)$ are calibrated for all relevant market factors affecting contractual cash flows and, to some extent, option exercise decisions. A market risk premium is implied from the prices of actively-traded and liquid hedging instruments.

On the other hand, exogenous or individual risk factors cannot easily be hedged but rather diversified away in a large portfolio. In this case, the traditional risk-adjusted pricing, followed, for example, by insurance companies, seems more appropriate. Firstly, the return of an instrument or portfolio is simulated under the physical measure $(P)$. Secondly, the expectation of future discounted cash flows is computed. Thirdly, a prudential risk charge is applied. Typically, this is equal to the remuneration on the risky capital which has to be set apart as a compensation for unexpected losses. Capital absorption is computed by means of a coherent risk measure and the premium is proportional to a hurdle rate for shareholders.

\footnotetext{
${ }^{4} \mathrm{~A}$ similar issue characterizes credit modeling, where we distinguish between default probabilities estimated at a particular time ("point-in-time" perspective) from their historical averages ("through-the-cycle" perspective). A data-set spanning over a long interval of time is needed to perform a robust calibration.
} 
In practice, we proceed as follows: $(i)$ we select a specific market scenario $X$; $(i i)$ we apply the risk charge approach to the conditional cash flow distribution; (iii) finally, we average across all possible market scenarios. Summing up, the price of a generic contract $V(t)$ with embedded options can be expressed by the following general formula

$$
\begin{aligned}
V(t) & =V_{E}(t)-V_{U}(t) \\
V_{E}(t) & =\mathbb{E}_{X}^{Q}\left[\mathbb{E}_{Z, \tau}^{P}[\Psi \mid X]\right] \\
V_{U}(t) & =k \cdot \mathbb{E}_{X}^{Q}\left[\Phi_{Z, \tau}^{P}[\Psi ; q \mid X]\right]
\end{aligned}
$$

where $\Phi$ is a coherent risk measure, $q$ is a target quantile and $k$ is the unitary cost of capital.

The first term, $V_{E}(t)$, corresponds to the expectation of discounted cash flows, $\Psi$, under a risk-neutral probability measure, where behavioral risk is costless $(k=0)$ or completely diversified $\left(\Phi^{P}=0\right)$.

The second term, $V_{U}(t)$, is the premium required by a risk averse investor to bear behavioral risk. It can also be interpreted as the potential extra-cost necessary to compensate for unavoidable hedging errors of a replicating strategy based on instruments, whose price is affected by market risk factors only.

In line with XVA methodology, it is possible to introduce the concept of behavioral value adjustment $\beta$ VA. For a review of the most widespread value adjustments refer, e.g., to Brigo et al. [23].

From the option seller's viewpoint, the price of a contract with embedded options can be written in the general form

$$
V(t)=\mathbb{E}_{X, Z, \tau}^{Q}[\Psi]=V_{H}(t)-\mathrm{OVA}(t)+\beta \mathrm{VA}(t)
$$

where

- $V_{H}(t)$ is the price of the host instrument, i.e. the contract without any prepayment option;

- OVA $(t)$ is the option value adjustment corresponding to the full rational cost of the option;

- $\beta \mathrm{VA}(t)$ is the behavioral value adjustment quantifying the potential benefit for the option seller that the option holder does not take advantage of. 
Behavioral risk adjustments $\beta$ VA can be split as follows:

$$
\begin{aligned}
\beta \mathrm{VA}(t) & =\beta \mathrm{VA}_{E}(t)-\beta \mathrm{VA}_{U}(t) \\
\beta \mathrm{VA}_{E}(t) & =\mathbb{E}_{X}^{Q}\left[\mathbb{E}_{Z, \tau}^{P}[\Psi \mid X]\right]-V_{H}(t)+\operatorname{OVA}(t) \\
\beta \operatorname{VA}_{U}(t) & =k \cdot \mathbb{E}_{X}^{Q}\left[\Phi_{Z, \tau}^{P}[\Psi ; q \mid X]\right]
\end{aligned}
$$

The two components, $\beta \mathrm{VA}_{E}(t)$ and $\beta \mathrm{VA}_{U}(t)$, quantify the expected gain and the potential unexpected losses due to the uncertain sub-optimal exercise strategy followed by the option holder, respectively.

Finally, an appropriate and coherent risk measure, $\Phi^{P}$, in (7) has to be chosen. Typically, one can select expected shortfall (ES) relative to the present value of future cash flows through the entire life of the contract. Unfortunately, this may require time-consuming Monte Carlo simulations of all risk factors.

In order to improve analytical tractability and speed up calculations, if the distribution is not excessively skewed, we can adopt an analytical risk measure, linked to the conditional standard deviation.

$$
\Phi^{P}(\Psi \mid X)=\chi_{q} \cdot \sqrt{\mathbb{V}_{Z, \tau}^{P}[\Psi \mid X]}
$$

where $\chi_{q}$ is a scaling factor which depends on the shape of the distribution and a quantile $q$. As a result, formula (5) can be rewritten as

$$
V(t)=\mathbb{E}_{X}^{Q}\left[\mathbb{E}_{Z, \tau}^{P}[\Psi]-k \cdot \chi_{q} \cdot \sqrt{\mathbb{V}_{Z, \tau}^{P}[\Psi]} \mid X\right]
$$

\subsection{A general formula for portfolio valuation}

The discounted payoff of a single mortgage can be expressed by the following formula ${ }^{5}$

$$
\Psi=\sum_{k=1}^{T} D_{k} \cdot C_{k} \cdot \mathbb{I}\left(\tau>t_{k}\right)+\sum_{k=1}^{T} D_{k} \cdot E_{k} \cdot \mathbb{I}\left(\tau=t_{k}\right)
$$

where $\tau$ is the exercise time and, for each date $k$,

- $D_{k}$ is the aleatory discount factor

- $C_{k}$ is the contractual cash flow if the option is not exercised

- $E_{k}$ is the prepayment amount

\footnotetext{
${ }^{5}$ For the sake of simplicity and consistent with standard numerical pricing algorithms, we assume that early exercise can occur at a discrete set of dates, like in Bermudan-style prepayment options.
} 
In the case of level payment mortgages without prepayment penalties, $C_{k}$ is constant and equal to the installment amount, while $E_{k}$ coincides with the outstanding balance of the loan. As a consequence, $D$ and $\tau$ are the only aleatory variables. The former depends on market factors only, while the latter is also affected by exogenous factors.

The payoff can be rewritten in terms of survival indicator functions with simple passages:

$$
\Psi=\sum_{k=0}^{T} D_{k} \cdot M_{k} \cdot \mathbb{I}\left(\tau>t_{k}\right)
$$

where

$$
\begin{cases}M_{0}=E_{1} \cdot D_{1} & \text { with } D_{0}=1, \mathbb{I}\left(\tau>t_{0}\right)=1 \\ M_{T}=C_{T}-E_{T} & \\ M_{k}=C_{k}-E_{k}+E_{k+1} \cdot D_{k+1} / D_{k} & \text { for } 0<k<T\end{cases}
$$

We consider now a portfolio of mortgages, issued continually at different times and with different contractual conditions. Let us define:

- $N$, the total number of mortgage debtors

- $M$, the number of mortgage contract types (i.e. same issue time, maturity, coupon rate and frequency, etc.)

- $N^{p}$, the number of contracts of type $p$

- $N^{i p}$, the number of contracts of type $p$ closed with the $i$-th debtor $\left(\sum_{i=1}^{N} N^{i p}=N^{p}\right)$.

The discounted payoff of the portfolio can be written as

$$
\Psi=\sum_{i=1}^{N} \sum_{p=1}^{M} N^{i p} \cdot\left(\sum_{k=0}^{T} D_{k} \cdot M_{k}^{p} \cdot \mathbb{I}\left(\tau^{i p}>t_{k}\right)\right)
$$

By applying (13) and the law of total variance

$$
\mathbb{V}[\Psi(x, y)]=\mathbb{V}_{x}\left[\mathbb{E}_{y}[\Psi \mid x]\right]+\mathbb{E}_{x}\left[\mathbb{V}_{y}[\Psi \mid x]\right]
$$

we can derive a general pricing formula for the portfolio

$$
V(0)=\mathbb{E}_{X}^{Q}\left[\Pi_{0}(X)-k \cdot \chi_{q} \cdot \sqrt{\Pi_{1}(X)+\Pi_{2}(X)}\right]
$$


with

$$
\begin{aligned}
& \Pi_{0}(X)=\mathbb{E}_{Z}^{P}\left[\mathbb{E}_{\tau}^{P}\left[\sum_{i=1}^{N} \sum_{p=1}^{M} \sum_{k=0}^{T} \Psi_{k}^{i p} \mid X, Z\right] \mid X\right] \\
& \Pi_{1}(X)=\mathbb{E}_{Z}^{P}\left[\mathbb{V}_{\tau}^{P}\left[\sum_{i=1}^{N} \sum_{p=1}^{M} \sum_{k=0}^{T} \Psi_{k}^{i p} \mid X, Z\right] \mid X\right] \\
& \Pi_{2}(X)=\mathbb{V}_{Z}^{P}\left[\mathbb{E}_{\tau}^{P}\left[\sum_{i=1}^{N} \sum_{p=1}^{M} \sum_{k=0}^{T} \Psi_{k}^{i p} \mid X, Z\right] \mid X\right]
\end{aligned}
$$

where we have defined

$$
\Psi_{k}^{i p}=N^{i p} \cdot D_{k} \cdot M_{k}^{p} \cdot \mathbb{I}\left(\tau^{i p}>t_{k}\right)
$$

The first term, $\Pi_{0}(X)$, is the revised expectation of discounted cash flows, conditionally to a particular market scenario and including the impact of exogenous factors. It quantifies the expected benefit for the option seller due to the sub-optimal exercise strategy followed by the option holder.

The second term, $\Pi_{1}(X)$, can be interpreted as a sort of granularity adjustment. It reflects the variability of future cash flows, since the option exercise time $\tau$ is not uniquely determined by the market scenario but it can occur potentially at any time. However, in the limit of a granular portfolio 6 it tends to vanish and can be disregarded.

The third term, $\Pi_{2}(X)$, quantifies the additional uncertainty induced by exogenous factors, which alter exercise probabilities for the same market scenario. It becomes negligible in a large portfolio only if exogenous factors are purely idiosyncratic (no system component).

By applying condition (2), we obtain:

$$
\begin{aligned}
& \Pi_{0}(X)=\sum_{i=1}^{N} \sum_{p=1}^{M} \sum_{k=0}^{T} L_{k}^{i p} \cdot \Gamma_{k, i, p}^{0} \\
& \Pi_{1}(X)=\sum_{i=1}^{N} \sum_{p=1}^{M} \sum_{k, h=0}^{T} L_{k}^{i p} L_{h}^{i p} \cdot \Gamma_{i, k h, p}^{1} \\
& \Pi_{2}(X)=\sum_{i, j=1}^{N} \sum_{p, q=1}^{M} \sum_{k, h=0}^{T} L_{k}^{i p} L_{h}^{j q} \cdot \Gamma_{i j, k h, p q}^{2}
\end{aligned}
$$

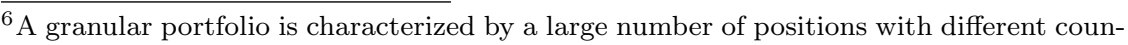
terparts and almost equal sizes.
} 
where we have defined

$$
\begin{aligned}
L_{k}^{i p} & =N^{i p} \cdot D_{k} \cdot M_{k}^{p} \\
\Gamma_{i, k, p}^{0} & =\mathbb{E}_{Z}^{P}\left[S_{k}^{i p} \mid X\right] \\
\Gamma_{i, k h, p}^{1} & =\mathbb{E}_{Z}^{P}\left[S_{\max (k, h)}^{i p} \mid X\right]-\mathbb{E}_{Z}^{P}\left[S_{k}^{i p} S_{h}^{i p} \mid X\right] \\
\Gamma_{i j, k h, p q}^{2} & =\mathbb{V}_{Z}^{P}\left[S_{k}^{i p}, S_{h}^{j q} \mid X\right]
\end{aligned}
$$

and $S_{k}^{i p}$ are survival probabilities, conditionally to a risk scenario $\{X, Z\}$. For each borrower, contract and possible exercise date

$$
S_{k}^{i p}(X, Z)\left\{\begin{array}{l}
=\mathbb{E}\left[\mathbb{I}\left(\tau^{i p}>t_{k}\right) \mid X, Z\right] \quad \forall k>0 \\
=1 \text { if } k=0
\end{array}\right.
$$

It is worth noting that the price depends only on the specification of the dynamics of both market and exogenous factors, as well as their functional dependence with survival probabilities $S_{k}^{i p}(X, Z)$.

\section{Mortgage portfolio valuation with BIX model}

In this section, we apply the general pricing framework described above to the valuation of a portfolio of mortgages, issued at different times and with different contractual rates, by Bissiri and Cogo [4], by extending pricing formulas to the case of a heterogeneous pool of mortgagors.

From the previous section we recognize that a particular model choice consists in: $(i)$ characterizing mortgagor creditworthiness and behavioral attitude to early prepayment; (ii) selecting all market and exogenous factors $\{X(t), Z(t)\} ;(i i i)$ specifying their dynamics; (iv) assuming a marginal probability response function, $R$, as defined in (1).

\subsection{Heterogeneity and granularity}

Firstly, heterogeneity in the pool is taken into account by dividing mortgagors into $C$ distinct clusters, with homogeneous degree of creditworthiness and prepayment attitude. ${ }^{7}$

We denote with $N^{c}$ the number of mortgagors in cluster $c$ and similarly with $N^{c p}$ the number of contracts of type $p$ owned by borrowers belonging

\footnotetext{
${ }^{7}$ We implicitly assume that the issuer has a good knowledge of the characteristics of all counterparts.
} 
to cluster $c$. Clearly,

$$
\begin{aligned}
\sum_{i=1}^{N} \mathbb{I}(i \in c) & =N^{c} & \sum_{c=1}^{C} N^{c} & =N \\
\sum_{i=1}^{N} \mathbb{I}(i \in c) \cdot N^{i p} & =N^{c p} & \sum_{c=1}^{C} N^{c p} & =N^{p}
\end{aligned}
$$

\subsection{Market factors}

Mortgage valuation is affected by interest and credit risk, besides behavioral risk. A sound and realistic model should capture the joint evolution of market interest rates and credits spread for all debtors. Several approaches can be chosen with increasing complexity. ${ }^{8}$

In this paper, for the sake of simplicity, we select a standard two-factor Gaussian model, belonging to the so-called family of intensity-based models, whose risk neutral dynamics is described by the stochastic processes

$$
\begin{gathered}
\left\{\begin{array}{c}
d X_{1}(t)=-\alpha_{1} \cdot d X_{1}(t) \cdot d t+\sigma_{1} \cdot d W_{1}(t) \\
d X_{2}(t)=-\alpha_{2} \cdot d X_{2}(t) \cdot d t+\sigma_{2} \cdot d W_{2}(t)
\end{array}\right. \\
\mathbb{E}^{Q}\left[d W_{1}(t) \cdot d W_{2}(t)\right]=\rho_{X} \cdot d t
\end{gathered}
$$

The first risk factor models essentially interest rates, while the second one accounts for default intensities for all mortgagors in the portfolio. Conditional to a specific risk factor scenario, defaults occur independently. A correlation between factors, $\rho_{X}$, is also introduced. See e.g. Brigo and Mercurio [20] or Schönbucher [21] for more details.

Due to the affine properties of the Gaussian models, interest rates and credit spreads, $Y(t)$, can be computed analytically

$$
Y(t)=a+b \cdot X_{1}(t)+c \cdot X_{2}(t)
$$

In particular, discount factors for cash flows of mortgages held by any debtor $i$ belonging to cluster $c$ are stochastic variables, expressed by

$$
D_{k}^{i}=D_{k}^{c}=\exp \left\{-\psi_{k}^{c}-\int_{0}^{t_{k}} X_{1}(u) d u-\int_{0}^{t_{k}} X_{2}(u) d u\right\} \forall i \in c
$$

where $\psi_{k}^{c}$ are deterministic shifts that account for the initial term structure of interest rates and credit spread for each cluster of borrowers.

\footnotetext{
${ }^{8}$ Credit spreads depend both on default probabilities and recovery rates. A full detailed model of mortgagor creditworthiness as well as the impact of collateral on recovery rates is beyond the scope of the present paper.
} 


\subsection{Exogenous factors}

We adopt a micro-structural (reduced-form) approach, by assigning a single (synthetic) exogenous factor to each single debtor in the portfolio. In order to capture additional correlation besides financial convenience, we assume that each factor can be split into a systemic component, $\xi_{k}^{0}$, and a purely idiosyncratic one, $\xi_{k}^{i}$.

$$
Z_{k}^{i}=Z^{i}\left(t_{k}\right)=\rho_{Z} \cdot \xi_{k}^{0}+\sqrt{1-\rho_{Z}^{2}} \cdot \xi_{k}^{i}
$$

where $\rho_{Z}$ is the weight of the systemic component, $\rho_{Z} \in[0,1]{ }^{9}$

In order to mimic fluctuations of observed prepayment rates around their historical mean, we also model the dynamics of each exogenous factor by means of an auto-regressive process, $\mathrm{AR}(1)$, with common parameters $(\alpha, \beta)$, so that

$$
\begin{aligned}
\xi_{k}^{i}= & \alpha \cdot \xi_{k-1}^{i}+\beta \cdot \varepsilon_{k}^{i} \\
\mathbb{E}_{Z}^{P}\left[\varepsilon_{k}^{i}\right]=0 ; \quad & \mathbb{E}_{Z}^{P}\left[\varepsilon_{k}^{i} \varepsilon_{h}^{j}\right]=\delta_{k h} \delta_{i j} \quad \forall i, j \geq 1, k, h>0
\end{aligned}
$$

We also assume that $\varepsilon_{0}^{i}=0 \forall i>0$, while $\varepsilon_{0}^{0}$ is a model parameter.

It is easy to compute that

$$
\begin{aligned}
\xi_{k}^{i} & =\lambda_{k, 0} \cdot \varepsilon_{0}^{i}+\sum_{h=1}^{k} \lambda_{k, h} \cdot \varepsilon_{h}^{i} \\
\lambda_{k, 0} & =\alpha^{k} ; \quad \lambda_{k, h}=\alpha^{k-h} \cdot \beta
\end{aligned}
$$

The exogenous process has to satisfy conditions (4) on the asymptotic distribution. When applied to the logarithm of $Q(t)$, model parameters are subject to the following constraints:

$$
\begin{aligned}
\mathbb{E}_{Z}^{P}\left[\xi_{\infty} \mid X\right]=0 & \Rightarrow|\alpha|<1 \\
\mathbb{V}_{Z}^{P}\left[\xi_{\infty} \mid X\right]=1 & \Rightarrow \beta=\sqrt{1-\alpha^{2}}
\end{aligned}
$$

\subsection{Marginal exercise probabilities}

Finally, we need to specify the dependence of marginal exercise probabilities on market and exogenous factors. In principle, a different response function $Q_{k}^{i p}$ can be calibrated for each mortgage with respect to contract type, loan age and mortgagor cluster. However, a large historical data-set

\footnotetext{
${ }^{9} \mathrm{~A}$ similar approach can be found in the Vasicek model, which is at the foundation for Basel standardized approach to credit risk measurement.
} 
would be needed to achieve a robust calibration. In practice, a simplified parametrization is more appropriate in most situations.

In the BIX model [4], $Q_{k}^{i p}$ at time $t_{k}$ for $i$-th owner of contract of type $p$ has the general form

$$
\ln \left[1-Q_{k}^{i p}\right]=A_{k}^{i p}(X)+B_{k}^{i p}(X) \cdot Z_{k}^{i}
$$

where $A$ and $B$ are generic functions. By taking cluster homogeneity into account, the following conditions hold

$$
A^{i p}=A^{c p}, B^{i p}=B^{c p} \quad \forall i \in c
$$

All response functions (45) have to be calibrated with historical prepayment data. For this purpose, it is convenient to select some representative market observables, $Y(t)$, such as interest rates and credit spreads. Thanks to the analytical properties of Gaussian models, their exact expression as a function of $X$ can easily be derived, so that

$$
\begin{aligned}
Y(t) & =\left\{Y_{1}(t, X(t)), Y_{2}(t, X(t)), \ldots\right\} \\
A_{k}^{i p}(X) & \rightarrow A_{k}^{i p}[Y(X)] \quad B_{k}^{i p}(X) \rightarrow B_{k}^{i p}[Y(X)]
\end{aligned}
$$

In order to capture the S-shaped dependence of prepayment rates on the market scenario $X, A$ can be selected among the family of logit-like functions, with a set of shape parameters for calibration. In contrast, $B$ can be assumed, to a first approximation, constant and independent on $X$.

\subsection{Hints for calibration}

In this paragraph we provide some hints for model calibration. Further details can be found in Bissiri and Cogo [4].

Undoubtedly, it is worth pointing out that a crucial role is played by the extent of the available data-set with time-series of historical prepayments. In practice, we follow a three-step procedure:

- We assume that risk neutral Q-dynamics for all market factors can be calibrated with the prices of quoted instruments. Such an assumption is quite reasonable when interest rate risk is concerned, but rather questionable for credit risk, since hedging instruments are not often liquid or traded. ${ }^{10}$ Nonetheless, because this paper

\footnotetext{
${ }^{10}$ Indeed, interest rate dynamics can be calibrated with the quotes of several liquid instruments such as deposits, swaps, swaptions, etc. In contrast, only a few singlename CDS are actively traded so that risk-neutral default probabilities cannot easily be
} 
focuses on behavioral risk, we assume that a term structure of interest rates and credit spreads is available for all borrowers and used to calibrate $\psi_{k}^{c}$ in formula (37) for each cluster.

- Formula (45), as a particular case of (3), has a financial interpretation. Function $A$ represents the long-term mean of prepayment rates subject to market scenario $X$, whilst function $B$ accounts for the amplitude of deviations induced by (unitary) exogenous factors. If aggregate prepayment rates of mortgage baskets are observed for a long enough period of time, the impact of exogenous factors tends to cancel out on average. Thus $A$ and be $B$ can be estimated by fitting historical prepayments as a function of market regressors. Basically, $A$ corresponds to the best fit line, while $B$ accounts for the residual variance. Typically, $A$ can be chosen among the family of logit or sigmoid functions of the rate shift in order to reproduce quite accurately the average S-shaped observed in historical data, see, e.g., prepayment rates reported in Davidson and Levin [1] or Peristiani [22]. The size of residuals is given by function $B$.

- Fit residuals can be explained in terms of latent exogenous risk factors, besides financial reasons. The time-evolution of $Z_{t}$ can be deduced by inverting formula (45). Model parameters in (39) can be calibrated by means of a maximum-likelihood-estimation algorithm (see, e.g., Hamilton [24]). A similar approach can be found in Kolbe and Zagst [17].

\subsection{Survival exercise probabilities}

It is also useful to derive an analytical expression for survival probabilities. For each mortgagor belonging to cluster $c$ and holding a contract of type $p$ we can write

$$
S_{k}^{i p}(X, Z)=\prod_{h=1}^{k}\left[1-Q_{h}^{i p}(X, Z)\right]=e^{W_{k}^{i p}(X, Z)}
$$

where, according to (45) and (46),

$$
W_{k}^{i p}(X, Z)=\sum_{h=1}^{k} A_{h}^{c p}(X)+\sum_{h=1}^{k} B_{h}^{c p}(X) \cdot Z_{h}^{i}
$$

inferred from the market. This is even more obvious when dealing with retail mortgagors. However, it is worth noting that in several cases, like residential mortgages, credit risk is remarkably mitigated by the presence of a collateral, i.e. the house property. 
With simple passages

$$
\begin{aligned}
W_{k}^{i p}(X, Z)= & \sum_{h=1}^{k} A_{h}^{c p}(X)+\sum_{h=1}^{k} B_{h}^{c p}(X) \cdot Z_{h}^{i} \\
= & \sum_{h=1}^{k} A_{h}^{c p}(X)+\sum_{h=1}^{k} B_{h}^{c p}(X) \cdot \rho \cdot\left(\lambda_{h, 0} \cdot \varepsilon_{0}^{0}+\sum_{l=1}^{h} \lambda_{h, l} \cdot \varepsilon_{l}^{0}\right) \\
& +\sum_{h=1}^{k} B_{h}^{c p}(X) \cdot \sqrt{1-\rho^{2}} \cdot\left(\sum_{l=1}^{h} \lambda_{h, l} \cdot \varepsilon_{l}^{i}\right)
\end{aligned}
$$

Summing up, we obtain

$$
W_{k}^{i p}(X, Z)=\sum_{h=1}^{k} F_{h}^{c p}(X)+\sum_{h=1}^{k} G_{h, k}^{c p}(X) \cdot\left(\rho \cdot \varepsilon_{h}^{0}+\sqrt{1-\rho^{2}} \cdot \varepsilon_{h}^{i}\right)
$$

where

$$
\begin{aligned}
F_{h}^{c p}(X) & =A_{h}^{c p}(X)+B_{h}^{c p}(X) \cdot \rho \cdot \lambda_{h, 0} \cdot \varepsilon_{0}^{0} \\
G_{h, k}^{c p}(X) & =\sum_{l=h}^{k} B_{l}^{c p}(X) \cdot \lambda_{l, h}
\end{aligned}
$$

For each $i \in c$ and conditionally to a market scenario, $W_{k}^{i p}$ are normal variables with mean and variance

$$
\begin{gathered}
\mu_{k}^{i p}(X)=\mu_{k}^{c p}(X)=\mathbb{E}_{Z}^{P}\left[W_{k}^{i p} \mid X\right]=\sum_{h=1}^{k} F_{h}^{c p}(X) \\
\sigma_{k}^{i p^{2}}(X)=\sigma_{k}^{c p^{2}}(X)=\mathbb{V}_{Z}^{P}\left[W_{k}^{i p} \mid X\right]=\sum_{h=1}^{k} G_{h, k}^{c p}{ }^{2}(X)
\end{gathered}
$$

\subsection{Portfolio pricing}

The price, $V(0)$, can be computed by using formulas derived in Section 3.4. We need to compute the three quantities in (24), (25) and (26):

$$
\begin{aligned}
& \Pi_{0}(X)=\sum_{i=1}^{N} \sum_{p=1}^{M} \sum_{k=0}^{T} L_{k}^{i p} \cdot \mathbb{E}_{Z}^{P}\left[S_{k}^{i p} \mid X\right] \\
& \Pi_{1}(X)=\sum_{i=1}^{N} \sum_{p=1}^{M} \sum_{k, h=0}^{T} L_{k}^{i p} L_{h}^{i p} \cdot \mathbb{E}_{Z}^{P}\left[S_{\max (k, h)}^{i p}-S_{k}^{i p} S_{h}^{i p} \mid X\right] \\
& \Pi_{2}(X)=\sum_{i, j=1}^{N} \sum_{p, q=1}^{M} \sum_{k, h=0}^{T} L_{k}^{i p} L_{h}^{j q} \cdot \mathbb{V}_{Z}^{P}\left[S_{k}^{i p}, S_{h}^{j q} \mid X\right]
\end{aligned}
$$


where

$$
L_{k}^{i p}=L_{k}^{i p}(X)=N^{i p} \cdot D_{k}^{i} \cdot M_{k}^{p}
$$

Given the sub-portfolio of mortgages of type $p$ held by borrowers in cluster $c$, let us define $L_{k}^{c p}(X)$ as the aggregate discounted cash flows exchanged at time $t_{k}$

$$
L_{k}^{c p}=L_{k}^{c p}(X)=\sum_{i \in c} N^{i p} \cdot D_{k}^{c} \cdot M_{k}^{p}=N^{c p} \cdot D_{k}^{c} \cdot M_{k}^{p}
$$

and $H^{c p}$ as the Herfindahl-Hirschman Index $(\mathrm{HHI})^{11}$

$$
H^{c p}=\sum_{i \in c}\left(\frac{N^{i p}}{N^{c p}}\right)^{2}
$$

\subsubsection{Expression for $\Pi_{0}(X)$}

By using (55) and (56), the conditional expectation $\Pi_{0}(X)$ can be computed as

$$
\begin{aligned}
\Pi_{0}(X) & =\sum_{p=1}^{M} \sum_{c=1}^{C} \sum_{i \in c} \sum_{k=0}^{T} L_{k}^{i p} \cdot \mathbb{E}_{Z}^{P}\left[e^{W_{k}^{i p}} \mid X\right] \\
& =\sum_{p=1}^{M} \sum_{c=1}^{C}\left[L_{0}^{c p}+\sum_{k=1}^{T} L_{k}^{c p} \cdot e^{\mu_{k}^{c p}+\frac{1}{2}\left(\sigma_{k}^{c p}\right)^{2}}\right]
\end{aligned}
$$

\subsubsection{Expression for $\Pi_{1}(X)$}

The second term $\Pi_{1}(X)$ is also linear with respect to the number of contracts and investors.

$$
\begin{aligned}
\Pi_{1}(X) & =\sum_{p=1}^{M} \sum_{i=1}^{N} \sum_{k, h=0}^{T} L_{k}^{i p} L_{h}^{i p} \cdot \mathbb{E}_{Z}^{P}\left[e^{\left.W_{\max (k, h)}^{i p}-e^{W_{k}^{i p}+W_{h}^{i p}} \mid X\right]}\right. \\
& =\sum_{p=1}^{M} \sum_{c=1}^{C} H^{c p} \cdot \sum_{k=0}^{T} L_{k}^{c p} \cdot I_{k}^{c p} \cdot e^{\mu_{k}^{c p}+\frac{1}{2}\left(\sigma_{k}^{c p}\right)^{2}}
\end{aligned}
$$

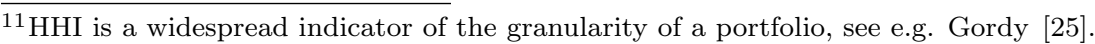


where $I_{k}^{c p}$ is approximated by ${ }^{12}$

$$
\begin{aligned}
I_{k}^{c p}=I_{k}^{c p}(X) \approx & L_{k}^{c p} \cdot\left(1-e^{\mu_{k}^{c p}+\frac{3}{2}\left(\sigma_{k}^{c p}\right)^{2}}\right) \\
& +2 \cdot \sum_{h=0}^{k-1} L_{h}^{c p} \cdot\left(1-e^{\mu_{h}^{c p}+\frac{1}{2}\left(\sigma_{h}^{c p}\right)^{2}+\sigma_{k}^{c p} \sigma_{h}^{c p}}\right)
\end{aligned}
$$

It is worth noting that $H^{c p}$ is usually vanishingly small in the granularity limit and, in turn, $\Pi_{1}(X)$ can be disregarded in large and equal-sized portfolios.

\subsubsection{Expression for $\Pi_{2}(X)$}

Finally, the computation for $\Pi_{2}(X)$ is less straightforward and more timeconsuming, due to the double loop on the number of contracts. Since it arises from conditional survival probability covariances, it becomes negligible only in the unrealistic case that exogenous risk is fully idiosyncratic.

Therefore, a different approach has to be followed with some suitable but accurate enough approximations.

- Firstly, we observe that $\Pi_{2}(X)$ is the variance of a sum of lognormal variables. Although an analytical expression for the resulting distribution is not available, we can apply one of the several approximations that have been derived in order to calculate analytically the first moments. ${ }^{13}$

- Secondly, the formula can be further simplified by assuming that the number of mortgagors with more than one position is relatively small if compared to the entire portfolio.

In this paper we adopt Gentle's approximation [26] by replacing the arithmetic weighted average with the geometric one, ${ }^{14}$ so that we can

\footnotetext{
${ }^{12}$ It can be shown that the following condition holds for $v_{h k}=\mathbb{V}\left[W_{k}+W_{h} \mid X\right]$. If $h<k, L_{1}<v_{h k}<L_{2}$ where $L_{1}=\sigma_{k}^{2}+3 \sigma_{h}^{2}$ and $L_{2}=3 \sigma_{k}^{2}+\sigma_{h}^{2}$. As a proxy, we take the geometric mean so that $v_{h k} \approx \sigma_{k}^{2}+\sigma_{h}^{2}+2 \sigma_{k} \sigma_{h}$. The impact of approximation has negligible impact especially if $\mu<<\sigma^{2}$ or $\sigma$ is almost constant as a function of time.

${ }^{13}$ For instance, this is the typical mathematical problem that one encounters in the pricing of equity Asian options in a Black-Scholes framework.

${ }^{14}$ Although several alternative and even more refined approximations have been developed, Gentle's approach is accurate enough as reported in the extensive literature about the pricing of Asian options.
} 
rewrite the expression for $\Pi_{2}(X)$ in the following way

$$
\begin{aligned}
\Pi_{2}(X) & =\mathbb{V}_{Z}^{P}\left[\sum_{p=1}^{M} \sum_{i=1}^{N} \sum_{k=1}^{T} L_{k}^{i p}(X) \cdot S_{k}^{i p}(X, Z) \mid X\right] \\
& \approx \mathbb{V}_{Z}^{P}\left[\prod_{p=1}^{M} \prod_{i=1}^{N} \prod_{k=1}^{T} e^{L_{k}^{i p}(X) \cdot W_{k}^{i p}(X, Z)} \mid X\right]=\mathbb{V}_{Z}^{P}\left[e^{\Omega(X, Z)} \mid X\right]
\end{aligned}
$$

where $\Omega(X, Z)$ is a normal random variable, with mean $M_{\Omega}(X)$ and variance $\Sigma_{\Omega}^{2}(X)$, respectively (see the Appendix (Section 6) for their analytical expressions). Thus, $\Pi_{2}(X)$ can be approximated with

$$
\Pi_{2}(X) \approx e^{2 \cdot M_{\Omega}(X)+\Sigma_{\Omega}^{2}(X)} \cdot\left(e^{\Sigma_{\Omega}^{2}(X)}-1\right)
$$

\subsection{Simulation}

Portfolio evaluation can be performed by means of Montecarlo simulations as in the full rational case. The only difference lies in the fact that, subject to a market scenario, cash flows are uniquely determined only if the option holder follows an optimal exercise strategy. In contrast, under the presence of behavioral risk, we need to compute the first two moments of the conditional distribution for each simulated path of market factors. However, we can rely on analytical formulas $(63),(64),(67)$. Since all formulas essentially depend on linear sums over the number of contracts and the number of dates, the additional computational burden is usually limited.

\section{Conclusion}

In conclusion, we have described a coherent and flexible framework for accounting the impact of behavioral risk on the valuation of a basket of mortgages. The approach consists in a prepayment model based on financial observables, which is needed to reproduce sub-optimal early prepayments in line with other models reported in the literature. At the same time, it introduces a pricing correction which accounts for the residuals that are observed when fitting historical data, as a consequence of the effect of exogenous factors. Due to their non-financial nature of these sources of risk, an additional adjustment is added to the pricing formula by adopting the traditional risk charge approach. We believe that our methodology is a valid and more transparent alternative to the practice of applying an option-adjusted-spread (OAS) to the discount factors in order to account for risk factors not capture by the financial prepayment model. 


\section{Appendix}

In this paragraph we derive an expression for the random variable $\Omega(X, Z)$, defined in (66), and we provide an analytical formula for its two conditional moments, $M_{\Omega}(X)$ and $\Sigma_{\Omega}^{2}(X)$.

$$
\begin{aligned}
& \Omega(X, Z)=\sum_{p=1}^{M} \sum_{k=1}^{T} \sum_{i=1}^{N} L_{k}^{i p}(X) \cdot W_{k}^{i p}(X, Z) \\
= & \sum_{k=1}^{T} \sum_{p=1}^{M} \sum_{c=1}^{C}\left[J_{k}^{c p}(X)+K_{k}^{c p}(X) \cdot\left(\rho_{Z} \cdot \varepsilon_{k}^{0}+\sqrt{1-\rho_{Z}^{2}} \cdot \sum_{i \in c}\left(\frac{N^{i p}}{N^{c p}}\right) \varepsilon_{k}^{i}\right)\right]
\end{aligned}
$$

where

$$
\begin{aligned}
J_{k}^{c p}(X) & =L_{k}^{c p}(X) \cdot \sum_{h=1}^{k} F_{h}^{c p}(X) \\
K_{k}^{c p}(X) & =\sum_{h=k}^{T} L_{h}^{c p}(X) \cdot G_{k, h}^{c p}(X)
\end{aligned}
$$

We can easily compute the conditional mean and variance of $\Omega(X, Z)$ as

$$
\begin{aligned}
& M_{\Omega}(X)=\mathbb{E}_{Z}^{P}[\Omega \mid X]=\sum_{k=1}^{T} \sum_{p=1}^{M} \sum_{c=1}^{C} J_{k}^{c p}(X) \\
& \Sigma_{\Omega}^{2}(X)=\mathbb{V}_{Z}^{P}[\Omega \mid X]=\sum_{k=1}^{T}\left[\rho_{Z}^{2} \cdot U_{k}^{1}(X)+\left(1-\rho_{Z}^{2}\right) \cdot U_{k}^{2}(X)\right]
\end{aligned}
$$

where

$$
\begin{aligned}
U_{k}^{1}(X) & =\left(\sum_{p=1}^{M} \sum_{c=1}^{C} K_{k}^{c p}(X)\right)^{2} \\
U_{k}^{2}(X) & =\sum_{c=1}^{C} \sum_{i \in c}\left(\sum_{p=1}^{M} \frac{N^{i p}}{N^{c p}} \cdot K_{k}^{c p}(X)\right)^{2}
\end{aligned}
$$

The expression can be further simplified in the quite realistic case where most mortgagors own a single contract, but contracts of a given type $p$ are owned by various mortgagors.

$$
U_{k}^{2}=\sum_{p=1}^{M} \sum_{c=1}^{C} H^{c p} K_{k}^{c p 2}
$$




$$
\Sigma_{\Omega}^{2}=\sum_{k=1}^{T}\left[\rho_{Z}^{2}\left(\sum_{p=1}^{M} K_{k}^{p}\right)^{2}+\left(1-\rho_{Z}^{2}\right) \sum_{p=1}^{M} \sum_{c=1}^{C} H^{c p} K_{k}^{c p 2}\right]
$$

\section{References}

1. A. Davidson and A. Levin, Mortgage Valuation Models: Embedded Options, Risk, and Uncertainty (Oxford University Press, 2014).

2. A. Castagna and F. Fede, Measuring and Managing Liquidity Risk (Wiley Finance, 2013).

3. M. Bissiri and R. Cogo, Modeling behavioral risk, Available at SSRN: https://ssrn. com/abstract=2523349 (2014).

4. M. Bissiri and R. Cogo, Behavioral Value Adjustments, Available at $S S R N$ : https://ssrn. com/abstract=2941815 (2017).

5. K. Dunn and J. McConnell, Valuation of GNMA mortgage-backed securities, Journal of Finance 36, 3 (1981), pp. 599-616.

6. R. Stanton, Rational Prepayment and the valuation of mortgagebacked securities, The Review of Financial Studies 8, 3 (1995), pp. $677-708$.

7. F. Longstaff, Borrower credit and the valuation of mortgage-backed securities, Real Estate Economics 33, 4 (2005), pp. 619-661.

8. A. Kalotay, D. Yang and F. Fabozzi, An option-theoretic prepayment model for mortgages and mortgage-backed securities, International Journal of Theoretical and Applied Finance 7, 8 (2004), pp. 949-978.

9. A. Davidson and A. Levin, Prepayment risk and option-adjusted valuation of MBS, The Journal of Portfolio Management 31, 4 (2005), pp. $73-85$.

10. E. S. Schwartz and W. N. Torous, Prepayment and the value of mortgage-backed securities, The Journal of Finance 44, 2 (1989), pp. 375-392.

11. Y. Deng, J. M. Quigley and R. Van Order, Mortgage terminations, heterogeneity, and the exercise of mortgage options, Econometrica 68, 2 (2000), pp. 275-307.

12. J. Kau, D. Keenan and A. Smurov, Reduced form mortgage pricing as an alternative to option-pricing models, The Journal of Real Estate Finance and Economics 33, 3 (2006), pp. 183-196.

13. Y. K. Kwok, M. Dai and H. You, Intensity-based framework and penalty formulation of optimal stopping problems, Journal of Economic Dynamics and Control 31, 12 (2007), pp. 3860-3880. 
14. M. Consalvi and G. Scotto di Freca, Measuring prepayment risk: an application to Unicredit Family Financing, Unicredit and Universities, Working paper series 5, (2010).

15. M. Chernov, B. Dunn and F. Longstaff, Macroeconomic-Driven Prepayment Risk and the Valuation of Mortgage-Backed Securities, $N a$ tional Bureau of Economic Research, Working paper 220968 (2016).

16. Y. Goncharov, An intensity-based approach to the valuation of mortgage contracts and computation of the endogenous mortgage rate, International Journal of Theoretical and Applied Finance 9, 6 (2006), pp. 889-914.

17. A. Kolbe and R. Zagst, A hybrid-form model for the prepayment-riskneutral valuation of mortgage-backed securities, International Journal of Theoretical and Applied Finance 11, 6 (2008), pp. 635-656.

18. A. Kolbe and R. Zagst, Valuation of mortgage-backed securities and mortgage derivatives: A closed-form approximation, Applied Mathematical Finance 16, 5 (2009), pp. 401-427.

19. X. Gabaix, A. Krishnamurthy and O. Vigneron, Limits of arbitrage: Theory and evidence from mortgage-backed-securities market, Journal of Finance 62, 2 (2007), pp. 557-595.

20. D. Brigo and F. Mercurio, Interest Rate Models: Theory and Practice with Smile, Inflation and Credit, 2nd edn. (Springer-Verlag, Berlin Heidelberg, 2006).

21. P. J. Schönbucher, Credit Derivatives Pricing Models (Wiley Finance, 2003).

22. S. Peristiani, Modeling the instability of mortgage-backed prepayments, The Journal of Fixed Income 13, 3 (2003), pp. 33-41.

23. D. Brigo, M. Morini and A. Pallavicini, Counterparty Credit Risk, Collateral and Funding: With Pricing Cases for all Asset Classes (Wiley Finance, 2013).

24. J. D. Hamilton, Time Series Analysis (Princeton University Press, 1994).

25. M. B. Gordy and E. Lütkebohmert, Granularity adjustment for Basel II, Deutsche Bundesbank Series 2, 1 (2007).

26. D. Gentle, Basket weaving, Risk 6 (1993), pp. 51-52. 